\title{
Perfil do consumo de álcool entre estudantes universitários*
}

Mariana Salles Motta Rodrigues de Barros ${ }^{1}$

Luciana Scarlazzari Costa²

O objetivo foi avaliar o perfil de consumo de bebidas alcoólicas entre estudantes de uma universidade brasileira. Quanto ao consumo de álcool, 76,6\% foram classificados com baixo risco e $23,4 \%$ de risco/uso abusivo. Os estudantes que faziam uso de tabaco e drogas ilícitas apresentaram riscos potencialmente elevados de consumo de álcool $\left(\mathrm{RP}_{\text {bruta }}=3,69, \mathrm{IC} 95 \%\right.$ : $2,01-6,79 ; \mathrm{RP}_{\text {bruta }}=2,44$, IC95\%: 1,32 - 4,50, respectivamente). Os estudantes apresentaram baixo risco de dependência, no entanto, os escores se mostraram diferenciados quando avaliado com características sociodemográficas e hábitos de vida.

Descritores: Consumo de Bebidas Alcoólicas; Alcoolismo; Estudantes; Psicotrópicos; Transtornos Relacionados ao Uso de Substâncias.

\footnotetext{
* Apoio financeiro da Fundação de Amparo à Pesquisa do Estado do Rio de Janeiro (FAPERJ), Brasil, processo $n^{\circ}$ E-26/100.622/2014.

1 Fundação Oswaldo Cruz, Rio de Janeiro, RJ, Brasil.

2 Universidade Metodista de Piracicaba, Piracicaba, SP, Brasil.
} 


\section{Alcohol consumption between students}

The aim of this study is to evaluate the profile of alcohol consumption among students of a Brazilian university. Regarding alcohol consumption, $76.6 \%$ are in the low risk of dependence and $23.4 \%$ in the risk/ harmful use. Students who used tobacco and illicit drugs presented potentially high risks of alcohol consumption ( $P R=3.69,95 \% \mathrm{Cl}$ : 2.01 to $6.79 ; \mathrm{PR}=2.44,95 \% \mathrm{Cl}$ : 1.32 to 4.50 , respectively). The students presented a low risk of alcohol dependence, however, scores consumption showed different when a comparison is made with sociodemographic characteristics and lifestyle habits.

Descriptors: Alcohol Drinking; Alcoholism; Students; Psychotropic Drugs; Substance-Related Disorders.

\section{Perfil del consumo de alcohol entre estudiantes universitarios}

El estudio tiene por objetivo evaluar el perfil de consumo de bebidas alcohólicas entre los estudiantes de una universidad brasileña. En relación con el patrón de consumo de alcohol, el $76,6 \%$ fue clasificado con bajo riesgo y el $23,4 \%$ en el riesgo/ abuso. Los estudiantes que hacían uso de tabaco y drogas ilícitas presentaron riesgos potencialmente elevados de consumo de alcohol $\left(R P_{\text {bruta }}=3,69\right.$; IC del 95\%: 2,01 a la 6,79; $R P_{\text {bruta }}=2.44$, IC del 95\%: 1.32 a la 4.50, respectivamente). Los estudiantes presentaron bajo riesgo de dependencia del alcohol, sin embargo, las puntuaciones de consumo se mostraron diferenciado cuando se evaluó con características sociodemográficas y hábitos vida.

Descriptores: Consumo de Bebidas Alcohólicas; Alcoholismo; Estudiantes; Psicotrópicos; Trastornos Relacionados con Sustancias.

Introdução

O álcool é uma substância psicotrópica que atua no sistema nervoso central provocando mudança no comportamento e potencial dependência em seus consumidores $^{(1)}$. No Brasil, essa substância tem sido de grande aceitação cultural em todas as classes sociais, e tem ampla disponibilidade comercial entre os jovens ${ }^{(2)}$.

Estudos epidemiológicos brasileiros estimaram elevadas prevalências pontuais de consumo de álcool, que variaram de $66,3 \%$ a $91,9 \%$, além disso, que o consumo abusivo de álcool é um grave problema de saúde pública no país ${ }^{(3-7)}$.

Quando se trata de jovens, o álcool é a droga mais consumida por eles no Brasil, seguida pelo tabaco, maconha e estimulantes; além de um importante fator na adoção de condutas de risco ${ }^{(8)}$. De acordo com o Centro Brasileiro de Informações sobre Drogas Psicotrópicas
(CEBRID)(1) $^{(1)} 73,2 \%$ dos jovens entre 18 e 24 anos já fizeram uso de bebidas alcoólicas alguma vez na vida e 15,5\% apresentam sintomas de dependência.

Pesquisas envolvendo o comportamento de universitários diante de substâncias psicoativas já foram feitos em universidades inter e nacionalmente. No Brasil, diversos estudos dentro desta temática já foram realizados com estudantes universitários ${ }^{(3-12)}$. A maior parte deles há a preocupação de se estudar o consumo entre alunos da área da saúde, como Ciências Biológicas, Medicina, Odontologia, Enfermagem, por serem estes os futuros formadores de opinião sobre saúde na sociedade. Nestes estudos há a preocupação em conhecer não somente a prevalência de consumo, mas também os possíveis fatores associados a esta prevalência.

Os fatores associados ao consumo de álcool entre estudantes são aqueles socioeconômicos, 
comportamentais e de relacionamento familiar. Dentre estes, pode-se destacar a renda, a escolaridade, a idade, o gênero, a cor da pele, a prática de religião, o estresse, a pressão do grupo, a prática ou não de esportes, trabalho, moradia longe da família, residência em áreas com poucas atividades de lazer, pouco diálogo com a família, problemas mentais, comportamento de risco entre outros ${ }^{(2,11-12)}$.

No Rio de Janeiro, com a população dos estudantes universitários observam-se estudos que envolvem o uso de drogas ou tabaco(13-14), e uso de uso de álcool(15-16). Estes estudos demonstram a preocupação e pertinência com o tema álcool e drogas, no contexto universitário, permitindo assim que estudos sejam conduzidos dentro desta temática de modo a se ampliar o conhecimento sobre ela. Assim, o objetivo deste trabalho é conhecer o perfil do consumo de álcool e fatores relacionados entre estudantes universitários em uma universidade pública do município do Rio de Janeiro, de acordo com características sociodemográficas e de hábitos de vida.

\section{Métodos}

O estudo foi do tipo observacional, transversal de abordagem quantitativa. Foi realizado no período de maio a outubro de 2013, participaram 124 alunos dos cursos de Biomedicina, Ciências Biológicas e Ciências da Natureza da Universidade Federal do Estado do Rio de Janeiro (UNIRIO). O projeto foi aprovado em 07/02/2013, CAAE: 03530412.3.0000.5285 pelo Comitê de Ética em Pesquisa da Universidade Federal do Estado do Rio de Janeiro.

O tamanho da amostra foi inicialmente avaliado pelo calculado amostral, estimando-se um total de 102 alunos, considerando uma proporção esperada de consumo de bebidas alcoólicas de 0,40, com intervalo de $95 \%$ de confiança, tamanho total 0,20 e percentual de perdas em torno de $10 \%$. A amostra total foi dividida considerando a proporção de cada curso no total de alunos atualmente matriculados nos quatro cursos (40\% de alunos em Biomedicina, 40\% em Biologia bacharelado, $8 \%$ em Ciências da natureza e $9 \%$ em Biologia licenciatura). Assim, foram incluídos os alunos, dos quatro cursos, que aceitaram participar da pesquisa.

A pesquisa foi realizada junto aos alunos nos intervalos entre as aulas no período de maio a outubro de 2013. Neste momento, foi avaliada apenas a idade do aluno, assim aos estudantes menores de dezoito anos, o termo de consentimento livre e esclarecido foi previamente enviado para que os pais/responsáveis. Posteriormente, aos participantes elegíveis um questionário com quatro partes foi utilizado. A saber:
(1) informações sociodemográficas, sobre consumo de álcool (2) Alcohol Use Disorder Identification TestAUDIT, (3) CAGE - Cut-down, Annoyed, Guilty e Eyeopener e sobre transtornos mentais (4) SQR-20 - Self Reporting Questionnaire. Todos os questionários foram autorrespondidos.

Para coletar as características sociodemográficas e de hábitos de vida foi utilizado um questionário específico para a pesquisa. Este apresenta questões sobre data de nascimento, sexo, cor da pele, escolaridade, curso, período, ano acadêmico, estado civil, religião, moradia, entre outras; e questões sobre uso de bebidas alcoólicas, hábito de fumar, uso de outras drogas e comportamento sexual. Para se obter a variável classe social utilizou-se o questionário do Critério de Classificação Econômica

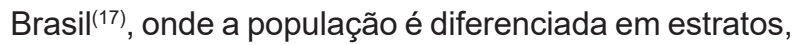
por suas características domiciliares, como presença de itens e a quantidade deles no domicílio.

O AUDIT (Alcohol Use Disorder Identification Test) é um questionário desenvolvido pela OMS, que rastreia o uso problemático de bebidas alcoólicas ${ }^{(18)}$. Foi adaptado para a população brasileira com boas qualidades psicométricas e utilizado em diversos estudos ${ }^{(19-20)}$.

O AUDIT é composto de dez questões, com respostas pontuadas. O escore varia de 0 a 40 pontos, e de acordo essa pontuação é possível identificar quatro diferentes padrões de consumo: baixo risco, que apresenta pontuação de 0 a 7; uso de risco, padrão de consumo que aumenta o risco de consequências negativas para quem consome e para quem está a sua volta, de 8 a 15; uso nocivo, aquele que pode levar a prejuízos físicos e mentais de 16 a 19; e provável dependência, 20 ou mais pontos, que é aquele consumo persistente apesar das consequências comportamentais, cognitivas e fisiológicas pelo uso repetido do álcool(18).

$\mathrm{Na}$ avaliação do presente estudo, considerou-se a nota do ponto de corte $\geq 8$ para o escore do AUDIT, caracterizado como uso problemático de álcool (uso de risco, uso nocivo e provável dependência)(18).

$\mathrm{Na}$ análise estatística, as variáveis qualitativas foram apresentadas por meio de porcentagens e as quantitativas por meio de estatística descritiva (média, mediana, desvio-padrão, mínimos e máximos). O padrão de consumo de álcool, ou seja, cada item do AUDIT foi descrito para os cursos avaliados e também para a amostra total. Realizou-se teste do qui-quadrado para verificar se o padrão de consumo difere em relação aos cursos. Além disso, o padrão de consumo de álcool foi analisado de duas maneiras.

Num primeiro momento, para avaliar a relação entre o escore de consumo e variáveis sociodemográficas e de hábitos de vida. O escore foi categorizado, obtendo-se os pontos de corte para os de baixo risco ou abstinentes 
(<8 pontos) e uso problemático ( $\geq 8$ pontos). Utilizou-se como medida de risco a razão de prevalência $(R P)$ e seu respectivo intervalo de confiança (IC). Num segundo momento, o escore de consumo foi avaliado como variável quantitativa e a sua descrição foi analisada por meio de estatística descritiva; a relação com as variáveis sociodemográficas e de hábitos de vida foi realizada por meio da comparação de médias. Para tanto, utilizou-se o teste não paramétrico de Wilcoxon.

Recursos gráficos como o diagrama de caixas (Box-Plot) também foram utilizados. Todas as variáveis sociodemográficas foram apresentadas na forma de variáveis qualitativas. Para efeitos de comparação, as variáveis quantitativas como idade e renda foram categorizadas em dois grupos, de acordo com a mediana dos valores. Para a variável renda utilizou-se o salário mínimo brasileiro para o ano de 2013, cujo valor era de $\mathrm{R} \$ 678,00$.

Em todas as análises considerou-se um a de 5\% e estas foram realizadas no $R$ versão 3.0.2.

\section{Resultados}

A amostra total foi de 124 alunos, matriculados nos seguintes cursos: Biologia bacharelado $(n=46)$ e licenciatura ( $n=13$ diurno e 4 do noturno), Biomedicina ( $n=51)$ e Ciências de Natureza $(n=10)$. Foram coletados os dados de 22 alunos a mais do que o número inicialmente proposto $(n=102)$.

Os estudantes foram caracterizados pelo sexo feminino, brancos, solteiros, morando com os pais, possuíam religião, sendo a maioria católica. A média de idade dos estudantes entrevistados foi de 21,3 anos (desvio-padrão=2,2 anos) e a renda média da família de $\mathrm{R} \$ 7.375,00$ (desvio-padrão=R $\$ 6.285,90$ ). A maioria é do curso de Biomedicina e pertence a da classe social $\mathrm{C}$. Os anos de 2010 e 2013 foram os períodos com maiores porcentagens de alunos ingressantes (Tabela 1).

Em relação aos hábitos de vida, observou-se que a maioria não era fumante e não usava droga ilícita, apesar dos casos alcoolismo na família. Apenas 15\% afirmaram que dirigiam sob o efeito de álcool e $26 \%$ fizeram sexo sem camisinha após ingerir bebidas alcoólicas (Tabela 1). A média de idade com que começaram a consumir álcool foi aos 15,2 anos (desvio-padrão=5 anos); a maioria deles relatou que não possuía hábito de experimentar bebidas alcoólicas após campanhas publicitárias e que também já presenciou campanhas contra o uso de álcool.

Tabela 1 - Descrição dos estudantes de acordo com as variáveis sociodemográficas e hábitos de vida. Rio de Janeiro, RJ, Brasil, dezembro de 2013

\begin{tabular}{cccc}
\hline Característica & Categoria & $\mathbf{n}$ & $\%$ \\
\hline Sexo & Feminino & 95 & 76,6 \\
Cor da pele & Branca & 94 & 75,8 \\
Estado Civil & Solteiro & 122 & 98,4 \\
Religião & Sim & 83 & 66,9 \\
Moradia & Com pais & 93 & 75,0 \\
Classe & A & 1 & 0,8 \\
& B & 23 & 18,5 \\
& C & 59 & 47,6 \\
& D & 34 & 27,4 \\
Curso & E & 7 & 5,7 \\
& Biomedicina & 51 & 41,1 \\
& C. Biológicas Bac. & 31 & 37,1
\end{tabular}




\begin{tabular}{|c|c|c|c|}
\hline Característica & Categoria & $\mathbf{n}$ & $\%$ \\
\hline \multirow[t]{2}{*}{ Uso de tabaco } & Sim & 41 & 25,0 \\
\hline & Não & 93 & 75,0 \\
\hline \multirow[t]{2}{*}{ Uso de droga ilícita } & $\operatorname{Sim}$ & 22 & 17,7 \\
\hline & Não & 102 & 82,3 \\
\hline \multirow[t]{2}{*}{ Casos de alcoolismo na família } & $\operatorname{Sim}$ & 64 & 51,6 \\
\hline & Não & 60 & 48,4 \\
\hline \multirow{2}{*}{$\begin{array}{l}\text { Uso de álcool após campanha publicitária } \\
\text { em favor }\end{array}$} & Sim & 35 & 28,2 \\
\hline & Não & 89 & 71,8 \\
\hline \multirow[t]{2}{*}{ Presenciou campanhas contra o álcool } & $\operatorname{Sim}$ & 87 & 70,2 \\
\hline & Não & 37 & 29,8 \\
\hline \multirow[t]{2}{*}{ Conhece efeitos do álcool } & $\operatorname{Sim}$ & 124 & 100,0 \\
\hline & Não & - & - \\
\hline \multirow[t]{2}{*}{ Dirigiu sobre o efeito do álcool } & $\operatorname{Sim}$ & 15 & 12,1 \\
\hline & Não & 109 & 87,9 \\
\hline \multirow[t]{2}{*}{ Conhece as DST's* } & Sim & 124 & 100,0 \\
\hline & Não & - & - \\
\hline \multirow[t]{2}{*}{ Fez sexo sem preservativo após beber } & Sim & 26 & 21,0 \\
\hline & Não & 98 & 79,0 \\
\hline Total & & 124 & 100 \\
\hline
\end{tabular}

${ }^{*}$ DSTs - Doenças Sexualmente Transmissíveis

Quanto a frequência do consumo de bebidas alcoólicas, 124 participantes, $42,7 \%$ referiram que consomem de 2 a 4 vezes por mês, $26,6 \%$ uma vez por mês ou menos, $9,7 \%$ duas a três vezes por semana e $0,8 \%$ quatro ou mais vezes por semana; $20,2 \%$ responderam que nunca consomem bebidas que contém álcool (dados não apresentados em Tabelas). Assim, a prevalência de consumo de bebidas alcoólicas foi de $79,8 \%$ na presente amostra.

De acordo com a classificação do AUDIT, 76,6\% (IC95\%: 69,0\% - 84,0\%) dos estudantes foram classificados como consumidores de baixo risco $(\leq 8)$, enquanto que $23,4 \%$ (IC95\%: $16,0 \%-31,0 \%$ ) foram classificados como bebedores problemáticos.

As variáveis associadas ao consumo de álcool foram o uso de tabaco (cigarro) e de drogas ilícitas (Tabela 2). A prevalência do consumo abusivo para os fumantes foi quase 4 vezes a dos não fumantes (IC95\%: 2,01-6,79), enquanto a prevalência do consumo abusivo de quem usa droga ilícita foi quase 3 vezes a prevalência de quem não a usa (IC95\%: 1,32 $-4,50)$. As demais variáveis não foram associadas com valores estatisticamente significativos com o consumo de álcool.

Tabela 2 - AUDIT, fatores sociodemográficos e hábitos de vida. Rio de Janeiro, RJ, Brasil, dezembro de 2013

\begin{tabular}{|c|c|c|c|c|}
\hline \multirow{3}{*}{ Variáveis } & \multirow{3}{*}{ Categoria } & \multicolumn{2}{|c|}{$\operatorname{AUDIT}^{*}(\mathrm{n} / \%)$} & \multirow{3}{*}{$\mathrm{RP}^{\dagger}(\mathrm{IC} 95 \%)$} \\
\hline & & $<8$ & $\geq 8$ & \\
\hline & & $95(\%)$ & $29(\%)$ & \\
\hline Sexo & Masculino vs feminino & $19(20,0)$ & $10(34,5)$ & $1,72(0,91-3,28)$ \\
\hline Cor da Pele & Branca vs não branca & $72(75,8)$ & $22(75,9)$ & $1,00(0,48-2,11)$ \\
\hline
\end{tabular}




\begin{tabular}{|c|c|c|c|c|}
\hline \multirow{3}{*}{ Variáveis } & \multirow{3}{*}{ Categoria } & \multicolumn{2}{|c|}{ AUDIT* (n/\%) } & \multirow{3}{*}{$\mathrm{RP}^{\dagger}(\mathrm{IC} 95 \%)$} \\
\hline & & $<8$ & $\geq 8$ & \\
\hline & & $95(\%)$ & $29(\%)$ & \\
\hline Religião & Não vs sim & $30(31,6)$ & $11(37,9)$ & $1,24(0,65-2,37)$ \\
\hline Moradia & Sem pais vs com pais & $23(24,2)$ & $8(27,6)$ & $1,14(0,56-2,31)$ \\
\hline Classe & $A+B$ vs $C+D+E$ & $17(17,9)$ & $7(24,1)$ & $1,33(0,64-2,74)$ \\
\hline Curso & Biomedicina vs outros & $37(38,9)$ & $14(48,3)$ & $1,34(0,71-2,52)$ \\
\hline Ano de ingresso & 2005 a 2009 vs 2010 a 2013 & $23(24,2)$ & $8(27,6)$ & $1,14(0,56-2,31)$ \\
\hline Uso de tabaco & Sim vs não & $15(15,8)$ & $16(55,2)$ & $3,69(2,01-6,79)$ \\
\hline Uso de droga & Sim vs não & $12(12,6)$ & $10(34,5)$ & $2,44(1,32-4,50)$ \\
\hline Álcool na família & Sim vs não & $48(50,5)$ & $16(55,2)$ & $1,15(0,61-2,19)$ \\
\hline $\begin{array}{l}\text { Bebeu após campanha } \\
\text { publ. }\end{array}$ & Sim vs não & $28(29,5)$ & $7(24,1)$ & $0,81(0,38-1,72)$ \\
\hline $\begin{array}{l}\text { Presenciou campanhas } \\
\text { contra }\end{array}$ & Sim vs não & $67(70,5)$ & $20(69,0)$ & $0,95(0,48-1,88)$ \\
\hline Dirigiu alcoolizad. & Sim vs não & $10(10,5)$ & $5(17,2)$ & $1,51(0,68-3,36)$ \\
\hline $\begin{array}{c}\text { Sexo sem preserv. após } \\
\text { beber }\end{array}$ & Sim vs não & $17(17,9)$ & $9(31,0)$ & $1,70(0,88-3,27)$ \\
\hline
\end{tabular}

* AUDIT: Alcohol Use Disorder Identification Test; † RP: Razão de Prevalência

Tabela 3 - Comparação dos escores do AUDIT*, de acordo com características sociodemográficas e de hábitos de vida. Rio de Janeiro, RJ, Brasil, dezembro de 2013

\begin{tabular}{|c|c|c|c|c|}
\hline \multirow{2}{*}{ Característica } & \multirow{2}{*}{ Categoria } & \multicolumn{2}{|c|}{ Escore AUDIT* } & \multirow{2}{*}{ p_valor } \\
\hline & & Média (dp) & Mediana (min-max) & \\
\hline Sexo & $\begin{array}{l}\text { Feminino } \\
\text { Masculino }\end{array}$ & $\begin{array}{l}4,4(4,1) \\
6,6(5,0)\end{array}$ & $\begin{array}{l}4,0(0,0-18,0) \\
6,0(0,0-19,0)\end{array}$ & 0,030 \\
\hline Cor da pele & $\begin{array}{c}\text { Branca } \\
\text { Não branca }\end{array}$ & $\begin{array}{l}4,8(4,3) \\
5,4(4,7)\end{array}$ & $\begin{array}{l}4,0(0,0-19,0) \\
4,0(0,0-18,0)\end{array}$ & 0,570 \\
\hline Estado Civil & $\begin{array}{c}\text { Solteiro } \\
\text { Não solteiro }\end{array}$ & $\begin{array}{l}5,0(4,4) \\
0,5(0,7)\end{array}$ & $\begin{array}{c}4,0(0,0-19,0) \\
0,5(0,0-1,0)\end{array}$ & 0,100 \\
\hline Religião & $\begin{array}{l}\text { Sim } \\
\text { Não }\end{array}$ & $\begin{array}{l}4,7(4,5) \\
5,4(4,2)\end{array}$ & $\begin{array}{l}4,0(0,0-19,0) \\
5,0(0,0-18,0)\end{array}$ & 0,216 \\
\hline Moradia & $\begin{array}{l}\text { Com pais } \\
\text { Sem pais }\end{array}$ & $\begin{array}{l}4,7(4,5) \\
5,6(4,3)\end{array}$ & $\begin{array}{l}4,0(0,0-19,0) \\
5,0(0,0-18,0)\end{array}$ & 0,211 \\
\hline Classe social & $\begin{array}{c}A+B \\
C+D+E\end{array}$ & $\begin{array}{l}6,0(4,9) \\
4,7(4,3)\end{array}$ & $\begin{array}{l}5,0(0,0-18,0) \\
4,0(0,0-19,0)\end{array}$ & 0,205 \\
\hline Curso & $\begin{array}{l}\text { Biomedicina } \\
\text { Ciências }\end{array}$ & $\begin{array}{l}5,0(4,9) \\
4,9(4,1)\end{array}$ & $\begin{array}{l}4,0(0,0-19,0) \\
4,0(0,0-18,0)\end{array}$ & 0,868 \\
\hline Ano de Ingresso & $\begin{array}{l}2009 \\
2005|-|-\mid \\
2010\end{array}$ & $\begin{array}{l}4,7(4,2) \\
5,0(4,5)\end{array}$ & $\begin{array}{l}4,0(0,0-14,0) \\
4,0(0,0-19,0)\end{array}$ & 0,803 \\
\hline Renda $^{\dagger}$ & $\begin{array}{l}<7,3 \mathrm{SM}^{\ddagger} \\
\geq 7,3 \mathrm{SM}^{\ddagger}\end{array}$ & $\begin{array}{l}3,7(4,2) \\
5,6(4,4)\end{array}$ & $\begin{array}{l}2,5(0,0-18,0) \\
5,0(0,0-19,0)\end{array}$ & 0,006 \\
\hline
\end{tabular}




\begin{tabular}{|c|c|c|c|c|}
\hline \multirow{2}{*}{ Característica } & \multirow{2}{*}{ Categoria } & \multicolumn{2}{|c|}{ Escore AUDIT* } & \multirow{2}{*}{ p_valor } \\
\hline & & Média (dp) & Mediana (min-max) & \\
\hline Uso de tabaco & $\begin{array}{l}\text { Sim } \\
\text { Não }\end{array}$ & $\begin{array}{l}8,3(4,7) \\
3,7(3,7)\end{array}$ & $\begin{array}{l}8,0(0,0-18,0) \\
3,0(0,0-19,0)\end{array}$ & $<0,001$ \\
\hline Uso de drogas ilícitas & $\begin{array}{l}\text { Sim } \\
\text { Não }\end{array}$ & $\begin{array}{l}8,0(4,6) \\
4,7(4,1)\end{array}$ & $\begin{array}{l}7,0(1,0-18,0) \\
3,5(0,0-19,0)\end{array}$ & $<0,001$ \\
\hline Alcoolismo na família & $\begin{array}{l}\text { Sim } \\
\text { Não }\end{array}$ & $\begin{array}{l}4,8(4,6) \\
5,0(4,3)\end{array}$ & $\begin{array}{l}4,0(0,0-18,0) \\
4,0(0,0-19,0)\end{array}$ & 0,587 \\
\hline Consumo após campanha & $\begin{array}{l}\text { Sim } \\
\text { Não }\end{array}$ & $\begin{array}{l}5,7(5,0) \\
4,7(4,2)\end{array}$ & $\begin{array}{l}5,0(0,0-19,0) \\
4,0(0,0-18,0)\end{array}$ & 0,350 \\
\hline Viu campanhas contra & $\begin{array}{l}\text { Sim } \\
\text { Não }\end{array}$ & $\begin{array}{l}5,1(4,6) \\
4,3(4,1)\end{array}$ & $\begin{array}{l}4,0(0,0-19,0) \\
3,0(0,0-18,0)\end{array}$ & 0,399 \\
\hline Dirigiu alcoolizado & $\begin{array}{l}\text { Sim } \\
\text { Não }\end{array}$ & $\begin{array}{l}7,2(4,5) \\
4,6(4,3)\end{array}$ & $\begin{array}{l}7,0(1,0-19,0) \\
4,0(0,0-18,0)\end{array}$ & 0,001 \\
\hline Sexo sem preservativo & $\begin{array}{l}\text { Sim } \\
\text { Não }\end{array}$ & $\begin{array}{l}7,3(4,8) \\
4,2(4,1)\end{array}$ & $\begin{array}{l}6,0(1,0-19,0) \\
3,0(0,0-18,0)\end{array}$ & $<0,001$ \\
\hline Escore total & & $4,9(4,4)$ & $4,0(0,0-19,0)$ & \\
\hline
\end{tabular}

*AUDIT: Alcohol Use Disorder Identification Test; †Renda em salário mínimo (SM=R \$678,00) referente ao ano de 2013 no Brasil; ¥SM - Salário Mínimo

$\mathrm{Na}$ comparação dos escores do AUDIT e as características sociodemográficas, foram observadas diferenças estatisticamente significativas para as variáveis: sexo masculino $(p=0,03)$ e renda $\geq R \$ 5000,00$ $(p=0,006)$ (Tabela 3).

Quando comparado os escores do AUDIT e os hábitos de vida, observam-se diferenças estatisticamente significativas para: uso de drogas ilícitas $(p<0,001)$ e uso de tabaco/cigarro $(p<0,001)$. Diferenças também foram observadas para as situações de dirigir alcoolizado $(p=0,001)$ e fazer sexo sem o uso de preservativos $(p<0,001)$ (Tabela 3).

O uso problemático de álcool foi maior entre os estudantes que possuíam hábitos como: uso de drogas ilícitas, cigarros, faziam sexo sem o uso de preservativos e dirigiam alcoolizados. Ainda, o risco de uso problemático de álcool aumentou consideravelmente entre quem fazia uso de substâncias e tinha comportamento de risco. A ilustração destes hábitos e comportamentos está na Figura 1.
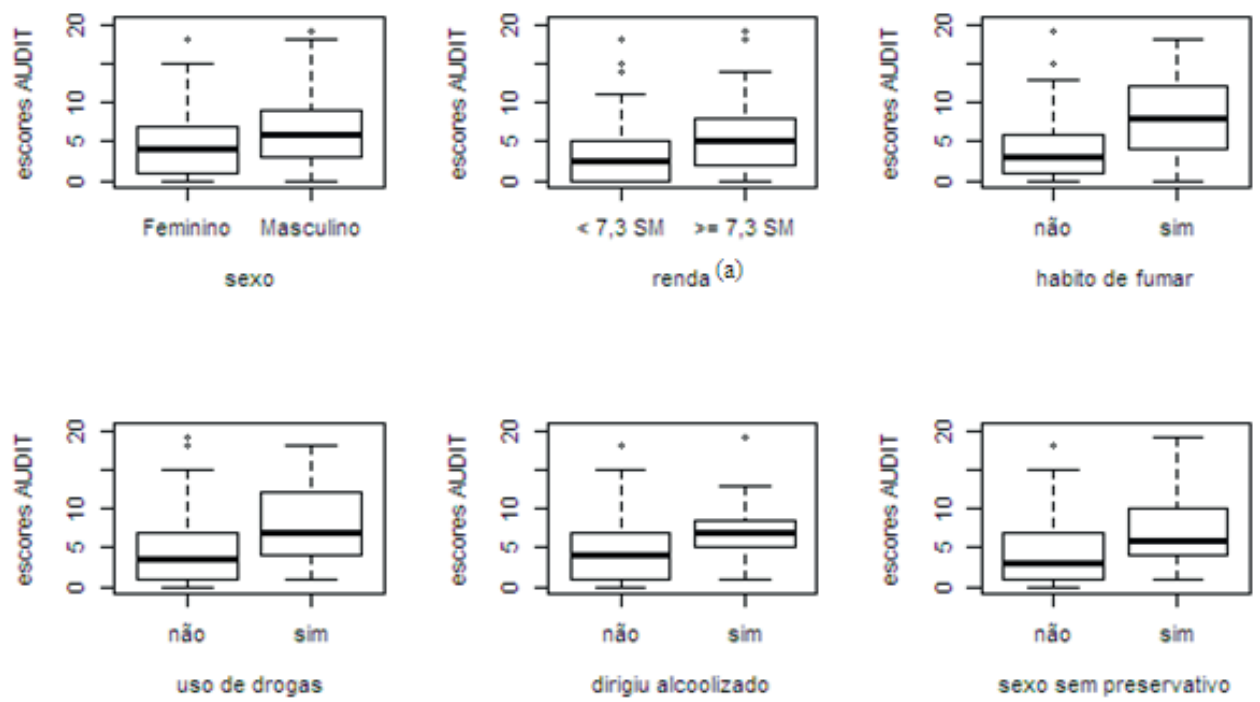

${ }^{(a)}$ Renda em salário minimo (SM=R $\left.\$ 678,00\right)$ referente ao ano de 2013 no Brasil.

Figura 1 - Distribuição dos escores do AUDIT de acordo com características sociodemográficas e hábitos de vida. Rio de Janeiro, RJ, Brasil, dezembro de 2013 


\section{Discussão}

Os estudantes se caracterizaram por serem mulheres, situações esta que se aproxima muito da proporção real de mulheres nos cursos estudados - Biomedicina, Biologia e Ciências da Natureza. A amostra inicialmente foi estratificada levando-se em consideração a proporção de alunos nos três cursos estudados, a qual se manteve após o levantamento de dados - Biomedicina $(41,1 \%)$, Biologia $(50,8 \%)$ e Ciências da Natureza $(8,1 \%)$.

A prevalência de consumo de álcool entre os estudantes da presente amostra foi alta, 79,8\% (IC95\%: 72\% - 87\%), o qual corrobora a do consumo de álcool da população brasileira $(86,2 \%)^{(20)} e$ também àquele encontrado em estudos realizadas com universitários ${ }^{(3,5,7,21)}$, apesar das diferenças metodológicas para se estimar o consumo. Com relação à periodicidade de consumo, 68,8\% dos estudantes relataram que bebem mensalmente ( 2 a 4 vezes ao mês). A média de idade com que os estudantes começaram a beber foi 15 anos, mesmo com a proibição por lei de consumo por menores de idade no país. Isso mostra que o hábito de beber foi anterior à entrada na faculdade, porém após o ingresso esse consumo pode ter ficado mais intenso e frequente ${ }^{(22)}$, devido a maiores oportunidades de interação e socialização entre os jovens, como em festas de faculdade e na ilusão de admiração e aceite pelo novo grupo (23-24).

Apesar da elevada prevalência de consumo, o consumo abusivo ou prejudicial do álcool foi de $23,4 \%$ para a amostra total de estudantes, enquanto que o uso de baixo risco foi de $76,6 \%$; estas proporções se mantiveram nos três cursos estudados. $O$ valor para o consumo abusivo corrobora ao encontrado entre estudantes de enfermagem $(20,5 \%)^{(25)}$, que utilizou a mesma metodologia para este desfecho e amostra semelhante no que se refere ao percentual de mulheres. Por outro lado, estudos nacionais com universitários, porém com outra metodologia para o desfecho, obtiverem prevalência menores de consumo abusivo $(8,7 \% \text { e } 14,3 \% \text {, respectivamente })^{(7,11)}$.

O consumo abusivo de álcool foi associado ao uso de tabaco, apresentando maior prevalência de risco entre os fumantes $(R P=3,69)$. A despeito das diferenças metodológicas, esta relação é corroborada pela literatura, que aponta maiores prevalências de consumo de álcool entre os tabagistas ${ }^{(13,26)}$.A prevalência de consumo de cigarro foi de $25 \%$, este valor se assemelha a de alunos das Ciências biológicas de São Paulo $(22,8 \%)^{(4)}$, mas é inferior aos valores encontrados em nacionais pregressos $(40 \% \text { a } 65 \%)^{(7)}$.
O uso de drogas ilícitas (maconha, crack, cocaína, alucinógenos, ecstasy e inalantes), embora com prevalência menor de consumo entre os estudantes $(17,7 \%)$, também esteve associado ao consumo de álcool, apresentando maiores prevalências de consumo abusivo para aqueles que faziam uso destas drogas $(R P=2,44)$. A porcentagem de alunos com uso problemático de álcool (AUDIT) também foi significativamente maior para os usuários de drogas ilícitas, de acordo com Silveira et al. ${ }^{(26)}$, os usuários de álcool tendem a entrar em contato com outras drogas. Isso pode refletir uma progressão natural de início em substâncias mais leves, como o álcool, avançando para outras mais pesadas a fim de conseguir novas sensações; ou os indivíduos fazem o uso múltiplo de substâncias como meio de controle, para aumentar os efeitos agradáveis ou diminuir os desagradáveis da outra droga ${ }^{(27-28)}$. No estudo de Andrade et al. (2010)(20), a maioria dos universitários começou a fazer uso de drogas ilícitas por curiosidade, e manteve esse hábito a fim de esquecer os problemas do dia a dia, seguidos por aqueles que usavam para controlar o efeito de outras substâncias.

Neste estudo, as prevalências de consumo abusivo de álcool não foram diferentes quando avaliadas de acordo com o sexo. No entanto, quando se observa os escores do AUDIT, houve maior consumo entre o sexo masculino, situação que reflete esta tendência do consumo maior entre os homens, devido às condições biológicas e culturais ${ }^{(7,11,21)}$.

No público de universitários do presente estudo, a renda parece permitir um maior acesso ao consumo de álcool, implicando assim em maiores níveis de consumo entre os estudantes com maior renda ( $R \$ \geq 5000)$. Tal relação foi descrita em estudos prévios, tanto por associação direta, do mesmo modo que o presente estudo, ou seja, indivíduos com maior renda consomem mais bebidas por maior poder de compra, e em outros estudos como inversa, ou seja, indivíduos com menos renda consomem mais, provavelmente para suprir a falta de atividades de lazer ${ }^{(11-12)}$.

Os estudantes que praticavam sexo sem o uso preservativo e dirigiam após beber apresentaram níveis problemáticos de consumo de álcool (AUDIT). Apesar disso, a totalidade dos estudantes relatou que conhecia os efeitos do álcool e também as doenças sexualmente transmissíveis. Os hábitos como beber e dirigir ou beber e praticar sexo inseguro são considerados comportamentos de alto risco, pois podem trazer consequências graves e indesejadas, como acidentes de trânsito e doenças sexualmente transmissíveis ${ }^{(21,26)}$. Estes comportamentos merecem atenção tanto por parte dos gestores, por meio do estabelecimento ou da 
intensificação dos programas de prevenção ao álcool e drogas, quanto por parte da família que tem papel fundamental na orientação destes jovens.

Com relação às limitações do estudo, este é um estudo transversal que avalia o consumo de álcool entre estudantes de uma universidade do Rio de Janeiro, e sendo assim ele não foi delineado para verificar possíveis relações causais entre fatores de risco e desfecho, nem tampouco para modelar o efeito independente dos fatores no consumo de bebidas alcoólicas. No entanto, os achados sobre associações aqui encontrados servem como ponto de partida para estudos futuros. Outra limitação deve-se ao fato de que a amostra foi não probabilística, pois se optou pela coleta dos dados em sala de aula, devido à facilidade de acesso aos estudantes. Considerando este horizonte, os resultados não devem ser generalizados para a realidade dos estudantes universitários, mas sim, para aqueles com o mesmo perfil de amostra aqui encontrada.

Estudos futuros com universitários merecem ser conduzido avaliando outros aspectos não abordados no presente estudo, como avaliação da qualidade de vida, estrutura familiar, estilo de vida e avaliação comportamental, visando ampliar a gama de fatores que podem estar relacionados ao consumo de álcool entre os estudantes. Estes estudos devem ser planejados com amostra probabilística e de modo a se levar em conta análises estratificadas e análises múltiplas.

\section{Conclusão}

Embora a prevalência de consumo de bebidas alcoólica tenha sido alta, a prevalência do consumo abusivo foi baixa entre os universitários dos cursos de Ciências Biológicas e da Natureza, e Biomedicina. Os alunos devem ser orientados com relação aos hábitos de vida como uso de drogas ilícitas, tabagismo, sexo desprotegido e o beber e dirigir, pois estes comportamentos estão muitas vezes relacionados ao consumo de bebidas alcoólicas entre os estudantes. Também é de grande valia que orientações sejam enfatizadas para os estudantes do sexo masculino com melhores níveis socioeconômicos.

\section{Referências}

1. Centro Brasileiro de informação sobre Drogas Psicotrópicas (CEBRID). II Levantamento Domiciliar sobre Uso de Drogas Psicotrópicas no Brasil, 2005. Disponível em: http://www.cebrid.epm.br. Acesso em: Dezembro de 2013.
2. Galduróz JCF, Sanchez ZM, Opaleye ES, Noto AR, Fonseca AM, Gomes PLS, et al. Fatores associados ao uso pesado de álcool entre estudantes das capitais brasileiras. Rev Saúde Pública. 2010; 44: 267-73.

3. Kerr-Corrêa F, Andrade AG, Bassit AZ, Boccuto NMVF. Uso de álcool e drogas por estudantes de medicina da Unesp. Ver Bras Psiquiatr. 1999; 21: 95-100.

4. Silva LVER, Malbergier A, Stempliuk VA, Andrade AG. Fatores associados ao consumo de álcool e drogas entre estudantes universitários. Rev Saúde Pública 2006; 40 (2): 280-8.

5. Franca C, Colares V. Comparative study of health behavior among college students at the start and end of their courses. Rev Saúde Pública. 2008; 42(3): 420-7.

6. Mardegan PS, Souza RS, Buaiz V, Siqueira MM. Uso de substâncias psicoativas entre estudantes de enfermagem. Rev Bras Psiquiatr. 2007; 56(4): 260-6.

7. Pedrosa AAS, Camacho LAB, Passos SRL, Oliveira RVC. Consumo de álcool entre estudantes universitários. Cad Saúde Pública. 2011; 27(8): 1611-1621.

8. Lucas ACS, Parente RCP, Picanço NS, Conceição DA, Costa KRC, Magalhães IRS et al. Uso de psicotrópicos entre universitários da área da saúde da Universidade Federal do Amazonas, Brasil. Cad Saúde Pública. 2006, 22(3): 663-671.

9. Paduani GF, Barbosa GA, Morais JCR, Pereira JCP, Almeida FM. Consumo de álcool e fumo entre os estudantes da Faculdade de Medicina da Universidade Federal de Uberlândia. Ver Bras Educ Med. 2008; 32: 66-74.

10. Chiapetti N, Serbena CA. Uso de álcool, tabaco e drogas por estudantes da área da saúde de uma universidade de Curitiba. Psicol Reflex Crít. 2007; 20(2): 303-13.

11. Dias-da-Costa JS, Silveira MF, Gazalle FK, Oliveira SS, Hallal PC, Menezes AMB, et al. Consumo abusivo de álcool e fatores associados: estudo de base populacional. Rev Saúde Pública. 2004; 38: 284-91.

12. Ferreira LN, Sales ZN, Casotti CA, Júnior JPB, Júnior ACRB. Perfil do consumo de bebidas alcoólicas e fatores associados em um município do Nordeste do Brasil. Cad. Saúde Pública. 2011; 27(8): 1473-1486.

13. Matsumoto KS, Craveiro MF, Rocha PR, Lopes GT. O uso do tabaco entre os universitários de enfermagem da universidade do estado do Rio de Janeiro (UERJ). Revista SMAD. 2005; 1(2): 1-14.

14. Passos SRL, Brasil PEAA, Santos MAB. Aquino MTC. Prevalence of psychoactive drug use among medical students in Rio de Janeiro. Soc Psychiatr Epidemiol. 2006; 41: 989-996.

15. Oliveira EB, Furegato ARF. O trabalho do acadêmico de enfermagem como fator de risco para o consumo de álcool e outras drogas. Rev. Latino-Am. Enfermagem. 2008; 16(especial): 565-71. 
16. Tavares-Jomar R, Santos-Silva E. Consumo de bebidas alcoólicas entre os estudantes de Enfermagem. Aquichan. 2013; 13 (2), 226-233.

17. Associação Brasileira de Empresas de Pesquisas - ABEP. Critério de Classificação Econômica do Brasil (CCEB), 2004. Disponível em: http://www.abep.org.br. Acesso em: Dezembro de 2013.

18. Babor, Thomas F, Higgins-Biddle, John C, Saunders, John B, Monteiro, Maristela G \& World Health Organization. Dept. of Mental Health and Substance Dependence. (2001)! AUDIT :the Alcohol Use Disorders Identification Test: guidelines for use in primary healthcare / Thomas $\mathrm{F}$. Babor...[et al.]; 2nd ed. Geneva: World Health Organization. Disponível em: http://www.who.int/iris/handle/10665/67205. Acesso em: Dezembro de 2013.

19. Moretti-Pires RO, Corradi-Webster CM. Adaptação e validação do Alcohol Use Disorder Identification Test (AUDIT) para população ribeirinha do interior da Amazônia, Brasil. Cad. Saúde Pública. 2011; 27(3): 497-509.

20. Andrade AG, Duarte PCAV, Oliveira LG. I Levantamento nacional sobre o uso de álcool, tabaco e outras drogas entre universitários das 27 capitais brasileiras - Secretaria Nacional de Políticas sobre drogas. Brasília; 2010.

21. Wagner GA, Andrade AG. Uso de álcool, tabaco e outras drogas entre estudantes universitários brasileiros. Rev Psiq Clín. 2008; 35 (suppl 1): 48-54.

22. Slutske WS. Alcohol use disorders among US college students and their non-college-attending peers. Arch Gen Psychiatry 2005; 62(3):321-7.

23. Borsari B, Carey KB. Peer influences on college drinking: a review of the research. J Subst Abuse. 2001; 13(4):391-424

24. Tapert S, Aarons G, Sedlar G, Brown S. Adolescent substance use and sexual risk-taking behavior. J Adolesc Health 2001; 28:181.

25. Pillon SC, Corradi-Webster CM. Teste de Identificação de consumo de álcool entre universitários. R Enferm UERJ. 2006; 14(3): 325-32.

26. Silveira CM, Silveira CC, Silva JG, Silveira LM, Andrade AG, Andrade LHSG. Epidemiologia do beber pesado e beber pesado episódico no Brasil: uma revisão sistemática da literatura. Rev Psiq Clín. 2008; 35(1): 31-38.

27. Hopper JW, Su Z, Looby AR, Ryan ET, Penetar DM, Palmer CM, Lukas SE. Incidence and patterns of polydrug use and craving for ecstasy in regular ecstasy users: an ecological momentary assessment study. Drug Alcohol Depend. 2006; 85(3):221-35.

28. Oliveira LG, Nappo SA. Characterization of the crack cocaine culture in the city of São Paulo: a controlled pattern of use. Rev Saude Publica. 2008;42(4):664-71. 